\section{Telephone book of life}

SIR — There are many different kinds of information systems such as libraries, computers, telephone books and the human genome. Would it help to understand the organization of the genome into coding and noncoding or 'junk' DNAs to the similarity of 'junk' DNA to information that we encounter every day in other information systems?

Familiar information systems have common characteristics. One common feature is that each information file occupies a unique location in threedimensional space, and access to a file is obtained by using codes. This means there is considerable access information contained in the architecture of the system itself. Think how difficult it would be to find a book in a library if all the books were piled in a large heap (even if they all had the proper catalogue numbers printed on them) or to find a telephone number if all the numbers were printed alphabetically on a single paper tape. Therefore, for ease of use, information systems usually consist mainly of the hardware architecture and access codes required to find information quickly and efficiently.

If we think of the cell nucleus as an information system consisting mainly of hardware (chromatin and nuclear architecture) and access codes (transactivator binding sites), then several characteristics of the nucleus and genome might become a bit more understandable because they may follow the same rules as other information systems.

A telephone book consists of hardware architecture in the arrangement of characters on its pages and of access codes for names and addresses. Because the only 'coding' information in the book is the telephone numbers themselves, any telephone book is composed mainly of noncoding sequences required to find those numbers - you don't use a telephone book to find names; you use the names to access telephone numbers. Promiscuous access motifs are found throughout any phone book - such as John, Mary, Dr, St or Ave - and they can mean different things depending on their location - as for Dr John Johnson of 63 Upjohn Dr.

To find hundreds of mindless repetitions of the same five-letter motif, one need only to go to Smith, A. and read down the left margin to Smith, $Z$. The access codes even work with multiple mismatches to a consensus spelling such as Chas or Jos. User-specific genes and pseudogenes are common in a telephone book - you get the numbers of all the butchers in town in your book even if you're a vegetarian and you get numbers for people who have died or moved away. Certainly the cell nucleus and the human genome it contains comprise 'the book of life'; not a novel to be read but rather a telephone book to provide correct information to individual users when they need it.

If anyone is uncomfortable with the idea of huge amounts of noncoding and junk DNA within the information storage medium of the human genome, I suggest four thought experiments which should indicate that noncoding and junk information are integral parts of any information system:

(1) Compile a complementary DNA library of a telephone book - to include only the telephone numbers (without the noncoding names and addresses next to them) that will be needed during the next year.

(2) Compile a personal computer floppy disk with all the information necessary for word processing, but where the 'noncoding' system and operating programs require less memory space than the 'coding' manuscript files.

(3) Scan the hard disk on your own personal computer for 'junk' - old or outdated manuscripts and data files that will probably never be used but that fill up the disk "just in case they may be useful some day'.

(4) Devise a logical method to scan the shelves in a library and remove all the books that will never be used in the lifetime of that library.

I believe that we can begin to obtain insights into the structure and function of the cell nucleus and human genome by assuming that it operates by rules similar to other information systems we use daily. If we look, we can see noncoding and junk information all around us.

John W. Bodnar

Chemistry Department,

US Naval Academy,

Annapolis,

Maryland 21402, USA

\section{European funds}

SIR - My impression of the European Commission (EC)'s Human Capital and Mobility programme is different from that of Lennart Philipson (Nature 360, 102 ; 1992). My experience is that of coordinating one of the Joint European Projects within the EC TEMPUS programme; although focused primarily on university teaching, it also has a large component of trans-European academic mobility.

The EC's recent involvement in funding of academic mobility has indeed completely changed the funding scene for European researchers. While fine- tuning of the procedures may be required, the Human Capital and Mobility programme is the best news European science has had for some time.

EC funding is available on a competitive basis to almost any centre within the European Communities. This is of particular interest to interdisciplinary scientists whose programmes might be judged to fall outside the scope of specialist organizations.

Moreover, in the past, more often than not, an award of a travelling fellowship has spelled financial disaster to a successful postdoctoral fellow. Better personal support will in itself assist the mobility of high-calibre researchers.

Inevitably, this new source of funds dwarfs in scale the present funding organizations. But nothing prevents individuals who are members of smaller organizations from applying for funding from the large EC programmes. Indeed, most people are familiar with a polite letter from the granting body saying "we hope you can obtain funding from another source".

Now, a realistic alternative source of funding exists. Notwithstanding procedural adjustments that still need to be made, it is certainly excellent news.

Marek H. Dominiczak

Department of Pathological Biochemistry, University of Glasgow,

Western Infirmary,

Glasgow G11 6NT, UK

\section{Centre move}

SIR - I believe that now is the appropriate time to reconsider and correct the term "Eastern Europe", often used in Nature.

This term was developed after 1945 to represent political rather than geographical realities in Europe and was used for countries under Soviet domination, despite their being in Europe. A few years ago the political situation changed drastically and many countries were liberated from the presently extinct Soviet Union.

The old term "Eastern Europe" now represents a nonexistent political situation in Europe and should be changed to a more precise geographical term. East Germany is no longer in Eastern Europe, while Poland, Czechoslovakia, Austria, Hungary and other countries of this region are Central European countries: Belorus, Ukraine and part of Russia are East European countries.

\section{J. Barankiewicz}

Gensia Pharmaceuticals, Inc., 11025 Roselle Street,

San Diego,

California 92121-1204, USA

$\square$ The point is well taken. - Editor, Nature. 